\title{
Efficacy of a CDK4/6 Inhibitor in a Patient with Breast Cancer and Liposarcoma: A Case Report and Review of the Literature
}

\author{
Laura Loretan $^{\mathrm{a}} \quad$ Linda Eszter Moskovszky $^{\mathrm{b}}$ Michael Kurrer ${ }^{c}$ G. Ulrich Exner ${ }^{\mathrm{d}}$ \\ Andreas Trojan ${ }^{\mathrm{e}}$ \\ aBrust-Zentrum Zürich, Zürich, Switzerland; \\ ${ }^{\mathrm{b}}$ Institut für klinische Pathologie, Universitätsspital Zürich, Zürich, Switzerland; \\ 'Gemeinschaftspraxis Pathologie Zürich, Zürich, Switzerland; \\ dOrthopädie Zentrum Zürich, Zürich, Switzerland: \\ e OnkoZentrum Zürich, Zürich, Switzerland
}

\section{Established Facts}

- Breast cancer is a heterogeneous disease and patients benefit from treatment with palbociclib and other CDK4/6 inhibitors.

- In clinical practice, the receptor status is used to justify treatment, which is limited to ER+/HER2breast cancer according to the current guidelines.

\section{Novel Insights}

- A variety of biomarkers are currently being explored to predict response to palbociclib, addressing novel insights in pharmacodynamics and resistance to this compound.

- The therapeutic application of palbociclib is not only restricted to ER+/HER2- breast cancer and has been recently tested in various different cancer types.

\section{Keywords}

Aromatase inhibitors · Biomarker · Breast cancer $\cdot$ CDK4/6 inhibitor - Drug resistance $\cdot$ Endocrine therapy $\cdot$ HER2 / HER2/neu . Hormonal treatment $\cdot$ Liposarcoma $\cdot$ Palbociclib

\section{Summary}

Background: The cyclin D/cyclin-dependent kinase (CDK)4/6 inhibitor of the CDK4 (INK4)/retinoblastoma (Rb) pathway plays a crucial role in cell cycle progression. Selective CDK4/6 inhibitors specifically target a variety of tumors, with the main focus on hormone receptor(HR)-positive and human epidermal growth factor receptor 2(HER2)-negative breast cancer (BC). Case Re- port: We report on the efficacy of neoadjuvant palbociclib and letrozole application in a patient suffering from invasive estrogen receptor (ER)+/HER2- BC and concurrent well-differentiated and dedifferentiated liposarcoma (WD-DDLPS) of the thigh. Clinical and histological workup upon surgery revealed significant regressive changes in both the liposarcoma and the BC. The 24month follow-up shows no signs of disease. Conclusion: CDK4/6 inhibitors exhibit a high therapeutic potential, although reliable prognostic markers need to be identified.

(c) 2018 S. Karger AG, Basel

\section{KARGER}

(c) 2018 S. Karger AG, Basel 


\section{Introduction}

Upon estrogenic or mitogenic/oncogenic signaling, breast tumors frequently achieve a deregulated state of proliferation through modifications of the cell cycle checkpoint involving phosphorylation of the retinoblastoma tumor suppressor protein (pRB), which is controlled by cyclin-dependent kinase (CDK) 4 and CDK6 $[1,2]$. Selective CDK4/6 inhibitors in combination with endocrine therapies have demonstrated efficacy, predominantly in hormone receptor(HR)-positive and human epidermal growth factor receptor 2(HER2)-negative breast cancer (BC) at moderate toxicities. U.S. Food and Drug Administration(FDA)-approved palbociclib, ribociclib, and abemaciclib, in combination with antihormones, are currently being explored in neoadjuvant and adjuvant settings in estrogen receptor(ER)-positive early BC [3-5]. Palbociclib in combination with letrozole and fulvestrant resulted in improved progression-free survival (PFS) in patients with $\mathrm{ER}+\mathrm{BC}$ [5-13]; in combination with other agents, $\mathrm{CDK} 4 / 6$ inhibitors are also investigated in advanced settings and in triple-negative BC (TNBC) [14].

Antiproliferative effects of CDK4/6 inhibition have also been reported in human liposarcoma [15], which represents the most frequent sarcoma among adults [16]. Among the 3 common subtypes, well-differentiated and dedifferentiated liposarcomas (WDDDLPSs) may exhibit a highly variable prognosis $[16,17]$ despite remarkable genetic similarity according to the amplification of the chromosome 12 region that codes for CDK4 and MDM2 (murine double minute 2 homolog) and renders them rather sensitive to targeted therapeutics [18].

The rare coincidence of a WD-DDLPS and a locally advanced ER+ HER2- BC thus prompted us to initiate CDK4/6 blockade in the patient described below. Here, we report on the efficacy of neoadjuvant palbociclib and letrozole and review the potential impact of CDK4/6 inhibition in different types of cancer and the mechanism of resistance.

\section{Case Report}

In August 2016, a 53-year-old woman presented with a 17-cm mass of the left thigh and a $7-\mathrm{cm}$ lump in the left breast associated with palpable axillary lymphadenopathy. Extensive workup revealed a WD-DDLPS and a moderately differentiated invasive ER+ and HER2- BC of non-specific type. No distant metastases were found, except for positron emission tomography(PET)-positive supraclavicular lymph nodes which were deemed not accessible for biopsy, indicating clinical stage cT3, N3, M0 stage III invasive BC. Since the patient refused to receive chemotherapy at first, and therapy of the $\mathrm{BC}$ seemed to be of putative prognostic relevance, treatment with the aromatase inhibitor letrozole $2.5 \mathrm{mg} /$ day combined with palbociclib ( $125 \mathrm{mg} /$ day during 28 days) was initiated, with the intention of a neoadjuvant and presumably targeted therapy. The 4-week follow-up already revealed a $20 \%$ volume reduction of the sarcoma, while sonographic monitoring of the left breast and associated lymph nodes indicated a partial response according to Response Evaluation Criteria in Solid Tumors (RECIST). As palbociclib was continued, the patient underwent surgery of the thigh. Since R0 resection was performed, no adjuvant radiation therapy was indicated according to the guidelines [19]. Strikingly, histological workup of the resected sarcoma revealed wide areas of necrosis and infiltration with foamy macrophages (fig. 1a-d), indicating the efficacy of CDK4/6 inhibition. After an-
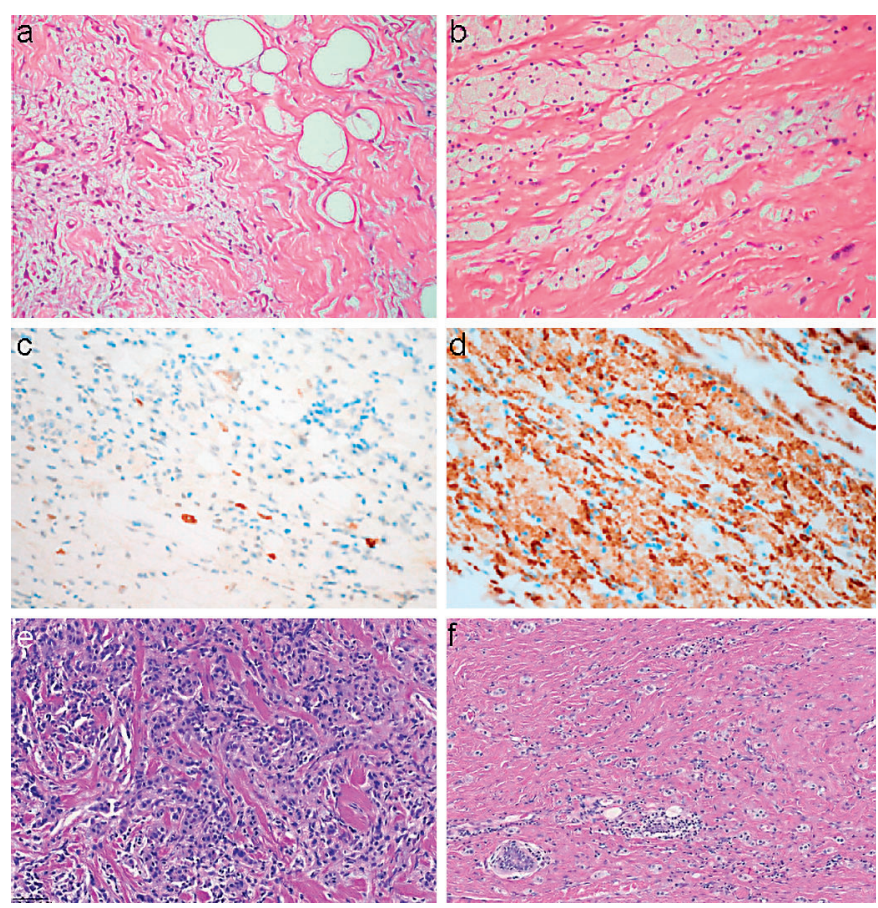

Fig. 1. a High-power magnification of the core needle biopsy specimen: Sclerosing fibrous proliferation with fat cells and occasional atypical cells with hyperchromatic nuclei, diagnostic of well-differentiated sclerosing liposarcoma. b Marginal parts of the resection specimen show sheets of histiocytic foam cells, indicating regressive changes in this liposarcoma after CDK4 inhibitor therapy. Immunohistochemical stains show c occasional tumor cells with nuclear positivity for MDM2 and $\mathbf{d}$ sheets of histiocytic foam cells with cytoplasmic positivity for CD68 (all $20 \times$ magnification). e High-power magnification (hematoxylin and eosin, $40 \times$ ) of a breast biopsy specimen at diagnosis with invasive breast carcinoma of non-specific type, infiltrating between preexisting glandular structures, and $\mathbf{f}$ showing regressive changes (fibrotic scar, elastic stromal degeneration, low tumor cellularity) after neoadjuvant therapy.

other 4 weeks of dual blockade with letrozole and palbociclib, surgery of the breast and axillary lymph nodes was performed. Although presurgical imaging had indicated a good partial remission of both the tumor (about 75\%) and lymph nodes, pathological workup identified tumor residues in 6 of 14 lymph nodes as well as highly regressive $\mathrm{BC}$ islets in a fibrotic area of $3.5 \mathrm{~cm}$ in diameter (fig. 1e, f), indicating a ypT1c, ypN2 post-neoadjuvant stage. Upon institutional tumor board recommendations, the patient could be convinced to continue therapy with 4 cycles of post-surgical epirubicin/cyclophosphamide chemotherapy, and adjuvant radiation (54 Gy) of the left breast and axillary, supra-infraclavicular and internal mammary lymph nodes was administered until March 2017. The patient also continued letrozole, intended for at least 7 years of duration. The current 24-month follow-up imaging reveals no evidence of disease. Of note, the described therapeutic approach resulted from a highly individual-based and not evidence-based decision and off-label use of palbociclib.

\section{Discussion and Review of the Published Literature}

The approval of palbociclib for treatment-naive ER+/HER2BC was based on PALOMA 1-3 study results, which demonstrated clinically meaningful benefits and improved rates of pathological response in pretreated settings $[7,8,20]$ but also in peri- or pre- 
menopausal women who additionally had received goserelin [21] Also a low incidence of grade 3-4 infections and febrile neutropenia had occurred; moreover, dose modifications to manage asymptomatic neutropenia did not obviously compromise the effect of palbociclib [5]. In neoadjuvant settings (e.g., the NeoPalAna trial), the rate of complete cell cycle arrest (CCCA) proved to be significantly higher with the addition of palbociclib to anastrozole in newly diagnosed ER+/HER2- BC (87 vs. 26\%) regardless of the luminal subtype (A vs. B) and the PIK3CA status (PIK3CA = phosphatidylinositol-4,5-bisphosphate 3-kinase catalytic subunit alpha) $[22,23]$. Neoadjuvant quadruple blockade with trastuzumab, pertuzumab, palbociclib, and fulvestrant also was found to be synergistic in terms of objective response rates in ER+/HER2+ BC patients (NA-PHER2 trial) [24]. Translational research has further explored a time- and dose-dependent inhibitory growth effect of palbociclib [25], with gene expression profiles [26] rendering the luminal ER+ subtype as most sensitive towards palbociclib, possibly related to the increase of $\mathrm{Rb}$ and cyclin $\mathrm{D}$ after estrogenic signaling and associated with a decrease in CDKN2A (CDK inhibitor $2 \mathrm{~A}$, p16) [27]. However, downregulation of cyclooxygenase 2 (COX-2) and prostaglandin E2 (PGE2) upon palbociclib treatment might induce antiproliferative and antimetastatic effects in both $\mathrm{ER}+$ and ER- BC [28]. In TNBC, combining doxorubicin with palbociclib seemed to induce cytostatic rather than cytotoxic effects in $\mathrm{Rb}$-proficient tumors [4]. However, variable effects might arise from CDK4/6 inhibition in these tumors, depending on, e.g., the androgen receptor status or a basal-like and mesenchymal subtype, potentially due to redundant cyclin E1 levels and high Rb phosphorylation [29].

In WD-DDLPS, a highly prevalent gene amplification or overexpression of CDK4, which leads to a maximized $\mathrm{Rb}$ phosphorylation, renders CDK4/6 inhibition a targeted therapy, although longterm exposure to ribociclib may cause resistance due to cyclin D1-3 accumulation $[15,17,18]$. Since WD-DDLPS frequently exhibits amplification of MDM2 and receptor tyrosine kinases (RTKs), concurrent MDM2 and CDK4 inhibition is thus expected to promote high rates of apoptosis and improve PFS [16, 30]. Synergistic effects may also encounter TP53 antiproliferative and proapoptotic activity, which is downregulated by MDM2 [30], and targeting of the frequently amplified RTKs such as the insulin-like growth factor 1 receptor (IGF1R) for clinical efficacy [16].

\section{Biomarkers and Resistance to Palbociclib}

To date, the clinical indication for CDK4/6 inhibitors is determined by the ER status [1]. Although clinically effective in the cohort of HER2+ BC, translational biomarker assessment identified neither Rb expression, Ki-67 and p16 loss nor cyclin D1 gene (CCND1) amplification as predictive for clinical benefit from palbociclib [11, 23, 27]. A chronic loss of $\mathrm{Rb}$ may be associated with the evolution to a CDK4/6-independent state, and ultimately resistance to palbociclib, but Rb-proficient tumors show no differ- ences in response to palbociclib when naively treated or being retreated $[23,26]$.

A comprehensive $\mathrm{Rb}$ loss signature ( $\mathrm{Rb}$-sig) consisting of 87 genes rather confirms that Rb-sig is able to differ between palbociclib-resistant and -sensitive tumors [23, 31]. A composite signature consisting of genes prone to CDK4/6 inhibition by palbociclib and abemaciclib did likewise [27], and, e.g., phosphorylation at T172 in the CDK4/cyclin D complex was associated with BC molecular subtypes and clinical efficacy [1].

Primary and acquired resistance to CDK4/6 inhibitors, however, seem to occur by bypassing the G1/S checkpoint through accumulation of a cyclin D1/CDK2 complex, which phosphorylates $\mathrm{Rb}$, or an increase of Akt phosphorylation, reflected by higher expression of E2F-dependent genes like cyclin E2 or CDK2. Potentially, an incomplete inhibition of $\mathrm{Rb}$ phosphorylation may be overcome by dual CDK4/6 and phosphoinositide 3-kinase (PI3K) blockade [23, 32], which might even be applicable in PI3K-mutated TNBC [33]. In how far 3-phosphoinositide-dependent protein kinase 1 (PDK1), downstream of PI3K, and CDK2 inhibitors will contribute to drug sensitivity and response or acquired resistance in $\mathrm{BC}$ and other cancers needs to be explored in translational research $[23,32,34]$.

Finally, the phenomena of autophagy and degradation of reactive oxygen species (ROS) in BC upon CDK4/6 low-dose inhibition was recently suspected as causative for resistance to palbociclib. Whether autophagy inhibitors such as hydroxychloroquine will significantly contribute to efficacy and tolerability of palbociclib in, e.g., Rb+/LMWE- TNBC (LMWE = low-molecular-weight cyclin E) and other solid tumors currently remains unclear [25]. With respect to WD-DDLPS, a variable drug efficacy due to the expression of MDM2 and a binding domain for p53 is reported. Loss of MDM2 may thus convert the quiescent to the senescent state and be associated with improved outcome [35]. Consequently, combination of the MDM2 inhibitor (RG7388) with palbociclib demonstrated a significantly longer PFS in animal experiments [30].

\section{Conclusions}

Drug development offers a great potential for the treatment of both CDK4- and Rb-proficient common and rare cancers. Despite impressive clinical efficacy, three limitations remain in the use of CDK4/6 inhibitors: lack of reliable biomarkers, development of resistance or adaption, as well as potential adverse events [25]. CDK4/6 inhibitors are now being clinically tested in a variety of other solid tumors, including glioblastoma [36, 37], hepatocellular carcinoma [38], and head and neck cancers, e.g. in combination with cetuximab [39].

\section{Disclosure Statement}

There are no potential conflicts of interest. 


\section{References}

1 Raspé E, Coulonval K, Pita JM, et al: CDK4 phosphorylation status and a linked gene expression profile predict sensitivity to palbociclib. EMBO Mol Med 2017;9:1052-1066.

2 Ingham M, Schwartz GK: Cell-cycle therapeutics come of age. J Clin Oncol 2017;35:2949-2959.

3 Trapé AP, Liu S, Cortes AC, Ueno NT, Gonzalez-Angulo AM: Effects of CDK4/6 inhibition in hormone receptor-positive/human epidermal growth factor receptor 2-negative breast cancer cells with acquired resistance to paclitaxel. J Cancer 2016;7:947-956.

$\checkmark 4$ McClendon AK, Dean JL, Rivadeneira DB, et al: CDK4/6 inhibition antagonizes the cytotoxic response to anthracycline therapy. Cell Cycle 2012;11:27472755.

5 Verma S, Huang Bartlett C, Schnell P, et al: Palbociclib in combination with fulvestrant in women with hor mone receptor-positive/HER2-negative advanced metastatic breast cancer: detailed safety analysis from a multicenter, randomized, placebo-controlled, phase III study (PALOMA-3). Oncologist 2016;21:1165-1175.

6 Finn RS, Crown JP, Lang I, et al: The cyclin-dependent kinase 4/6 inhibitor palbociclib in combination with letrozole versus letrozole alone as first-line treatment of oestrogen receptor-positive, HER2-negative, advanced breast cancer (PALOMA-1/TRIO-18): a randomised phase 2 study. Lancet Oncol 2015;16:25-35.

7 Cristofanilli M, Turner NC, Bondarenko I, et al: Fulvestrant plus palbociclib versus fulvestrant plus placebo for treatment of hormone-receptor-positive, HER2-negative metastatic breast cancer that progressed on previous endocrine therapy (PALOMA-3): final analysis of the multicentre, double-blind, phase 3 randomised controlled trial. Lancet Oncol 2016;17: 425-439.

8 Finn RS, Martin M, Rugo HS, et al: Palbociclib and letrozole in advanced breast cancer. N Engl J Med 2016;375:1925-1936.

9 Walker AJ, Wedam S, Amiri-Kordestani L, et al: FDA approval of palbociclib in combination with fulvestrant for the treatment of hormone receptor-positive, HER2-negative metastatic breast cancer. Clin Cancer Res 2016;22:4968-4972.

10 Turner NC, Ro J, André F, et al: Palbociclib in hormone-receptor-positive advanced breast cancer. N Engl J Med 2015;373:209-219.

11 DeMichele A, Clark AS, Tan KS, et al: CDK 4/6 inhibitor palbociclib (PD0332991) in Rb+ advanced breast cancer: phase II activity, safety, and predictive biomarker assessment. Clin Cancer Res 2015;21:9951001.

12 Finn RS, Crown JP, Ettl J, et al: Efficacy and safety of palbociclib in combination with letrozole as first-line treatment of ER-positive, HER2-negative, advanced breast cancer: expanded analyses of subgroups from the randomized pivotal trial PALOMA-1/TRIO-18. Breast Cancer Res 2016;18:67.
Harbeck N, Iyer S, Turner N, et al: Quality of life with palbociclib plus fulvestrant in previously treated hormone receptor-positive, HER2-negative metastatic breast cancer: patient-reported outcomes from the PALOMA-3 trial. Ann Oncol 2016;27:1047-1054.

14 Kwapisz D: Cyclin-dependent kinase 4/6 inhibitors in breast cancer: palbociclib, ribociclib, and abemaciclib. Breast Cancer Res Treat 2017;166:41-54.

15 Zhang Y-X, Sicinska E, Czaplinski JT, et al: Antiproliferative effects of CDK4/6 inhibition in CDK4-amplified human liposarcoma in vitro and in vivo. Mol Cancer Ther 2014;13:2184-2193.

16 Asano N, Yoshida A, Mitani S, et al: Frequent amplification of receptor tyrosine kinase genes in well-differentiated/dedifferentiated liposarcoma. Oncotarget 2017;8:12941-12952.

17 Dickson MA, Tap WD, Keohan ML, et al: Phase II trial of the CDK4 inhibitor PD0332991 in patients with advanced CDK4-amplified well-differentiated or dedifferentiated liposarcoma. J Clin Oncol 2013;31:20242028.

18 Dickson MA, Schwartz GK, Keohan ML, et al: Progression-free survival among patients with well-differentiated or dedifferentiated liposarcoma treated with CDK4 inhibitor palbociclib: a phase 2 clinical trial. JAMA Oncol 2016;2:937-940.

19 Ray-Coquard I, Thiesse P, Ranchère-Vince D, et al: Conformity to clinical practice guidelines, multidisciplinary management and outcome of treatment for soft tissue sarcomas. Ann Oncol 2004;15:307-315.

20 Witzel I, Müller V: The role of CDK 4/6 inhibitors in breast cancer treatment. Breast Care 2016;11:165-166.

21 Loibl S, Turner NC, Ro J, et al: Palbociclib combined with fulvestrant in premenopausal women with advanced breast cancer and prior progression on endocrine therapy: PALOMA-3 results. Oncologist 2017;22: 1028-1038.

22 Ma CX, Gao F, Luo J, et al: NeoPalAna: Neoadjuvant palbociclib, a cyclin-dependent kinase $4 / 6$ inhibitor, and anastrozole for clinical stage 2 or 3 estrogen receptor positive breast cancer. Clin Cancer Res 2017;23: 4055-4065.

23 Guarducci C, Bonechi M, Boccalini G, et al: Mechanisms of resistance to CDK4/6 inhibitors in breast cancer and potential biomarkers of response. Breast Care 2017;12:304-308.

24 Gianni L, Bisagni G, Colleoni M, et al: Neoadjuvant treatment with trastuzumab and pertuzumab plus palbociclib and fulvestrant in HER2-positive, ER-positive breast cancer (NA-PHER2): an exploratory, open-label, phase 2 study. Lancet Oncol 2018;19:249-256.

25 Vijayaraghavan S, Karakas C, Doostan I, et al: CDK4/6 and autophagy inhibitors synergistically induce senescence in $\mathrm{Rb}$ positive cytoplasmic cyclin E negative cancers. Nat Commun 2017;8:15916.

26 Finn RS, Dering J, Conklin D, et al: PD 0332991, a selective cyclin D kinase $4 / 6$ inhibitor, preferentially inhibits proliferation of luminal estrogen receptor-positive human breast cancer cell lines in vitro. Breast Cancer Res 2009;11:R77.
7 Knudsen ES, Hutcheson J, Vail P, Witkiewicz AK: Biological specificity of CDK4/6 inhibitors: dose response relationship, in vivo signaling, and composite response signature. Oncotarget 2017;8:43678-43691.

28 Qin G, Xu F, Qin T, et al: Palbociclib inhibits epithelial-mesenchymal transition and metastasis in breast cancer via c-Jun/COX-2 signaling pathway. Oncotarget 2015;6:41794-41808.

29 Asghar U, Barr AR, Cutts R, et al: Single-cell dynamics determines response to $\mathrm{CDK} 4 / 6$ inhibition in triple negative breast cancer. Clin Cancer Res 2017;23:55615572.

30 Laroche-Clary A, Chaire V, Algeo M-P, Derieppe M-A, Loarer FL, Italiano A: Combined targeting of MDM2 and CDK4 is synergistic in dedifferentiated liposarcomas. J Hematol Oncol 2017;10:123.

31 Malorni L, Piazza S, Ciani Y, et al: A gene expression signature of retinoblastoma loss-of-function is a predictive biomarker of resistance to palbociclib in breast cancer cell lines and is prognostic in patients with ER positive early breast cancer. Oncotarget 2016;7:6801268022.

32 Herrera-Abreu MT, Palafox M, Asghar U, et al: Early adaptation and acquired resistance to CDK4/6 inhibition in estrogen receptor-positive breast cancer. 2017; 131:393-409.

33 Andreopoulou E, Kelly CM, McDaid HM: Therapeutic advances and new directions for triple-negative breast cancer. Breast Care 2017;12:21-28.

34 Jansen VM, Bhola NE, Bauer JA, et al: Kinome-wide RNA interference screen reveals a role for PDK1 in acquired resistance to CDK4/6 inhibition in ER-positive breast cancer. Cancer Res 2017;77:2488-2499.

35 Kovatcheva M, Liu DD, Dickson MA, et al: MDM2 turnover and expression of ATRX determine the choice between quiescence and senescence in response to CDK4 inhibition. Oncotarget 2015;6:8226-8243.

36 Parrish KE, Pokorny J, Mittapalli RK, Bakken K, Sarkaria JN, Elmquist WF: Efflux transporters at the blood-brain barrier limit delivery and efficacy of cyclin-dependent kinase 4/6 inhibitor palbociclib (PD0332991) in an orthotopic brain tumor model. J Pharmacol Exp Ther 2015;355:264-271.

37 Raub TJ, Wishart GN, Kulanthaivel P, et al: Brain exposure of two selective dual CDK4 and CDK6 inhibitors and the antitumor activity of CDK4 and CDK6 inhibition in combination with temozolomide in an intracranial glioblastoma xenograft. Drug Metab Dispos 2015;43:1360-1371.

38 Hsieh F-S, Chen Y-L, Hung M-H, et al: Palbociclib induces activation of AMPK and inhibits hepatocellular carcinoma in a CDK4/6-independent manner. Mol Oncol 2017;11:1035-1049.

39 Rieke DT, Klinghammer K, Keilholz U: Targeted therapy of head and neck cancer. Oncol Res Treat 2016;39: 780-786. 